\title{
Estratégia multimodal para prevenção da infecção da corrente sanguínea relacionada ao cateter venoso central: uma revisão integrativa
}

\author{
Multimodal strategy for prevention of central venous catheter-related \\ bloodstream infection: an integrative review
}

Alanna Gomes da Silva ${ }^{1}$, Adriana Cristina de Oliveira ${ }^{2}$

Silva AG, Oliveira AC. Estratégia multimodal para prevenção da infecção da corrente sanguínea relacionada ao cateter venoso central: uma revisão integrativa / Multimodal strategy for prevention of central venous catheter-related bloodstream infection: an integrative review. Rev Med (São Paulo). 2017 out.-dez.;96(4):271-7.

RESUMO: Objetivo: Analisar as produções científicas nacionais e internacionais que discorram sobre as estratégias multimodais para prevenção de infecção da corrente sanguínea relacionada ao cateter venoso central em unidade de terapia intensiva adulto. Métodos: Realizou-se uma revisão integrativa da literatura de artigos publicados nas bases de dados PubMed, Science Direct, Cochrane, Cinahl e Scopus, de 2011 a 2016. Resultados: Encontraram-se 10 artigos que em sua totalidade enfatizaram ações voltadas para os períodos pré-intervenção com preparo da instituição e diagnóstico situacional, avaliação de protocolos e rotinas das instituições em 90\% dos artigos; na fase de intervenção predominou o preparo dos profissionais distinguindo-se pelo método como palestras, workshops, grupos de discussão e simulação visando a etapa de inserção e manutenção do cateter, e no pós-intervenção em 100\% dos estudos foi avaliado o impacto das estratégias implementadas sobre as taxas de infecção da corrente sanguínea relacionada ao CVC e disponibilizados feedback dos resultados e relatórios de vigilância à toda equipe. Conclusão: Os estudos apresentaram tempos de intervenção variados entre si, porém todos registraram uma redução da infecção da corrente sanguínea relacionada ao cateter venoso central entre 40 e $87 \%$, sendo observada uma relação direta entre o tempo da intervenção e a porcentagem de redução das taxas de infecção.

Descritores: Infecções relacionadas a cateter; Cateteres; Unidade de terapia intensiva; Infecção hospitalar; Segurança do paciente.

\begin{abstract}
Objective: To analyze the national and international scientific productions that discuss the multimodal strategies for prevention of bloodstream infection related to the central venous catheter in an adult intensive care unit. Methods: It was carried out an integrative review of the literature of articles published in the databases PubMed, Science Direct, Cochrane, Cinahl and Scopus, from 2011 to 2016. Results: It was found 10 articles that in their totality emphasized actions aimed at the pre-intervention periods with institution preparation and situational diagnosis, evaluation of protocols and routines of the institutions in $90 \%$ of the articles; In the intervention phase, the preparation of the professionals was predominant, and the method, such as lectures, workshops, discussion groups and simulation aimed at insertion and maintenance of the catheter, was used in the intervention phase in $100 \%$ of the studies. Implemented on CVC-related bloodstream infection rates and feedback of results and surveillance reports to the entire team. Conclusion: The studies presented varied intervention times, but all reported a reduction in central venous catheter-related bloodstream infection between 40 and $87 \%$, with a direct relationship between the time of the intervention and the percentage of reduction of the Rates of infection.
\end{abstract}

Descriptors: Catheter-related infections; Intensive care units; Cross infection; Patient safety.

1. Universidade Federal de Minas Gerais, Belo Horizonte, MG, BR. Enfermeira. Mestre em enfermagem. E-mail: alannagomessilva@ gmail.com.

2. Universidade Federal de Minas Gerais (UFMG), Escola de Enfermagem, Departamento de Enfermagem Básica, Belo Horizonte, MG, BR. Enfermeira. Pós-Doutora pela Universidade de Nova York. Professora Associada. Orientadora. E-mail: acoliveira@gmail.com.

Endereço para correspondência: Avenida Alfredo Balena, 190. Santa Efigênia, Belo Horizonte, MG, BR CEP: 30130-100. 


\section{INTRODUÇÃO} $A_{\text {saúde (IRAS) são consideradas um grave }}^{\text {s infeccões relacionadas à assistência em }}$ problema de saúde pública, devido a sua alta prevalência e as consequências de ordem pessoal, econômica e social que repercutem tanto para os pacientes quanto para as instituições de saúde ${ }^{1}$.

Entre as IRAS, a infecção da corrente sanguínea relacionada ao cateter venoso central (CVC) é apontada, quase sempre, como a mais frequente em unidade de terapia intensiva (UTI), devido a condição clínica dos pacientes, utilização de procedimentos invasivos, período de internação prolongado, uso de imunossupressores e antimicrobianos e consequente colonização por microrganismos resistentes ${ }^{2-3}$.

Estima-se que 30.000 novos casos dessa infecção ocorram em UTI dos Estados Unidos a cada ano ${ }^{4}$. Na Europa, em 2014 a taxa foi 13,3 infecções por 1000 CVCdia $^{5}$. No Brasil, a sistematização dos dados epidemiológicos sobre a infecção da corrente sanguínea relacionada ao CVC em UTI iniciou em 2010. Assim, as taxas dessa infecção variaram entre 4,1 a 5,1 infecções a cada 1000 CVC-dia até o ano de $2015^{6}$ e a mortalidade em pacientes internados em UTI pode atingir até $69 \%$.

A infecção da corrente sanguínea relacionada ao CVC é considerada, na maioria dos casos, uma complicação prevenível para a segurança do paciente. Por isso, a utilização de boas práticas durante a inserção e manutenção desses cateteres deve ser incluída na prática clínica visando reduzir tal complicação ${ }^{8}$. Nesse sentido, a estratégia multimodal ou multifacetada de forma concomitante pode apresentar resultados mais efetivos comparados à adoção de medidas isoladas na prevenção dessas infecções ${ }^{9-10}$. Contudo, percebe-se ainda uma baixa adesão das instituições a essa estratégia ou conjunto de medidas, além de poucos estudos que avaliam o uso de várias medidas conjuntas visando impactar na redução das taxas da infecção da corrente sanguínea relacionada ao CVC em UTI.

Diante do exposto, objetivou-se analisar as produções científicas nacionais e internacionais que discorram sobre a estratégia multimodal para prevenção de infecção da corrente sanguínea relacionada ao CVC em UTI adulto.

\section{MATERIAIS E MÉTODOS}

Tratou-se de uma de uma revisão integrativa da literatura, cuja finalidade consiste em reunir e sintetizar as evidências disponíveis em artigos originais produzidos sobre o tema, com o intuito de aperfeiçoar o conhecimento científico e as práticas profissionais.

Para a condução da revisão adotou-se a estratégia PICO que se estrutura da seguinte forma: $\mathrm{P}=$ Paciente, $\mathrm{I}=$
Intervenção, $\mathrm{C}=$ Comparação e $\mathrm{O}=$ Outcomes (desfecho). Nessa estratégia a definição da pergunta foi o primeiro passo para a proposta da investigação. Assim, definiu-se como pergunta de pesquisa: o que as produções científicas apontam sobre a estratégia multimodal para prevenção de infecção da corrente sanguínea relacionada ao CVC em UTI adulto?

Posteriormente foi realizada uma busca na literatura, formação de banco de dados, análise dos estudos encontrados, interpretação dos dados e discussão dos resultados.

A busca foi realizada em periódicos publicados em inglês e português por meio das bibliotecas: Portal CAPES e Biblioteca Virtual em Saúde (BVS) e as bases de dados: PubMed, Science Direct, Cochrane, CINAHL e Scopus.

Realizou-se a busca de estudos originais publicados a partir de 2011 por ser o ano em que o Center for Disease Control and Prevention (CDC) publicou o Guideline for the Prevention of Intravascular Catheter-Related. Foram utilizados os descritores controlados (DeCs): infecções relacionadas a cateter, cateteres, unidade de terapia intensiva e infecção hospitalar. Como descritores não controlados: estratégia multimodal, estratégia multifacetada, cateter venoso central, prevenção e infecção da corrente sanguínea. Todos esses descritores foram utilizados isoladamente e em conjunto, com auxílio dos operadores booleanos - AND, OR, AND NOT.

Foram estabelecidos como critérios de inclusão: artigos originais que se referiam à implementação da estratégia multimodal para prevenção da infecção da corrente sanguínea relacionada ao CVC em UTI adulto, independente do método de pesquisa empregado, excluindo todos os demais.

A partir da associação entre todos os descritores controlados e não controlados, identificou-se 23 artigos, que após a leitura na íntegra foram reduzidos a 10 , devido a duplicação em bases diferentes, indisponibilidade integral e discordância com os critérios de inclusão do estudo. Realizou-se um fichamento dessas publicações com as seguintes informações: título, autor, ano de publicação, nível de evidência, medidas implementadas, duração da intervenção e taxas de infecção, para auxiliar na análise e interpretação dos resultados.

Os níveis de evidências foram caracterizados de forma hierárquica, dependendo da abordagem metodológica adotada, com base na categorização da Agency for Healthcare Research and Quality (AHRQ) dos EUA $^{11}$, classificada em seis níveis:

- Nível 1: evidências resultantes da meta-análise (estudos clínicos controlados e randomizados);

- Nível 2: evidências obtidas de estudos com delineamento experimental;

- Nível 3: evidências de estudos quase experimentais; 
- Nível 4: evidências de estudos descritivos (não experimentais) ou com abordagem qualitativa;

- Nível 5: evidências provenientes de relatos de caso ou de experiência;

- Nível 6: evidências baseadas em opiniões ou consensos de especialistas.

\section{RESULTADOS}

Conforme os critérios de inclusão, a amostra final foi constituída por 10 artigos distribuídos nas bases de dados: Science Direct (4/10) e PubMed (6/10). Quanto ao delineamento da pesquisa verificaram-se em $100 \%$ estudos de coorte, com nível de evidência 2, realizados na Austrália (1/10), Espanha (1/10), Estados Unidos (3/10), Emirados Árabes (1/10), Grécia (1/10), Índia (2/10) e Suíça (1/10), e publicados entre os anos de 2011 a 2016.

Em 100\% dos artigos pesquisados conduziram os resultados conforme os períodos: pré-intervenção, intervenção e pós-intervenção.

O período pré-intervenção foi constituído pelo preparo da instituição e diagnóstico situacional, nos quais avaliaram os protocolos e rotinas das instituições em $90 \%$ dos $\operatorname{artigos}^{(12-20)}$ e em $50 \%$ realizam análises retrospectiva das taxas de infecção da corrente sanguínea relacionadas ao $\mathrm{CVC}^{14,16,19-21}$. A duração da implantação dessa fase variou entre 3 a 36 meses.

O período de intervenção consistiu na implementação de bundles para prevenção da infecção da corrente sanguínea relacionada ao CVC. Desta forma, os profissionais responsáveis pela manutenção e inserção do cateter participaram de treinamentos sobre essa temática. A condução desses treinamentos foi diferente entre os estudos, alguns realizaram grupos de discussão ${ }^{12,18}$; workshop com palestras, vídeos dos procedimentos de inserção e cuidados com o CVC e simulação dessas práticas ${ }^{13,20}$; palestras e distribuição de cartilhas com as recomendações preconizadas $^{14-17,19,21}$.

As medidas implementadas durante o período de intervenção estavam relacionadas à inserção e manutenção do CVC e estão apresentadas a seguir:

Bundle de inserção do CVC: barreira máxima de precaução (uso de luva estéril, gorro, máscara, capote e campo estéril - $100 \%$ dos estudos); Assepsia da pele com clorexidina alcóolica (100\%); Remoção imediata do CVC sem indicação clínica para seu uso (80\%); higiene das mãos antes da inserção (70\%); Evitar a veia femoral (60\%); Check list de inserção (40\%); Preferência pela veia subclávia (30\%); kits exclusivos para inserção do CVC (30\%) e CVC impregnado com antibiótico (10\%).

Bundle de manutenção: verificação diária da necessidade de permanência do CVC (80\%); Higiene das mãos antes da manipulação (70\%); Desinfecção do hub antes de administrar medicamentos (40\%); Registro do tempo de permanência do CVC (30\%); Assepsia da pele com clorexidina alcóolica ao trocar o curativo (20\%); Banho diário com clorexidina (20\%); Substituição do curativo com gaze estéril a cada 2 dias e curativo transparente a cada 7 dias ou sempre que estivessem sujo, solto ou úmido (10\%) e curativos impregnados com antissépticos (10\%).

A frequência em que essas medidas foram descritas nos estudos estão apresentadas na Figura 1.

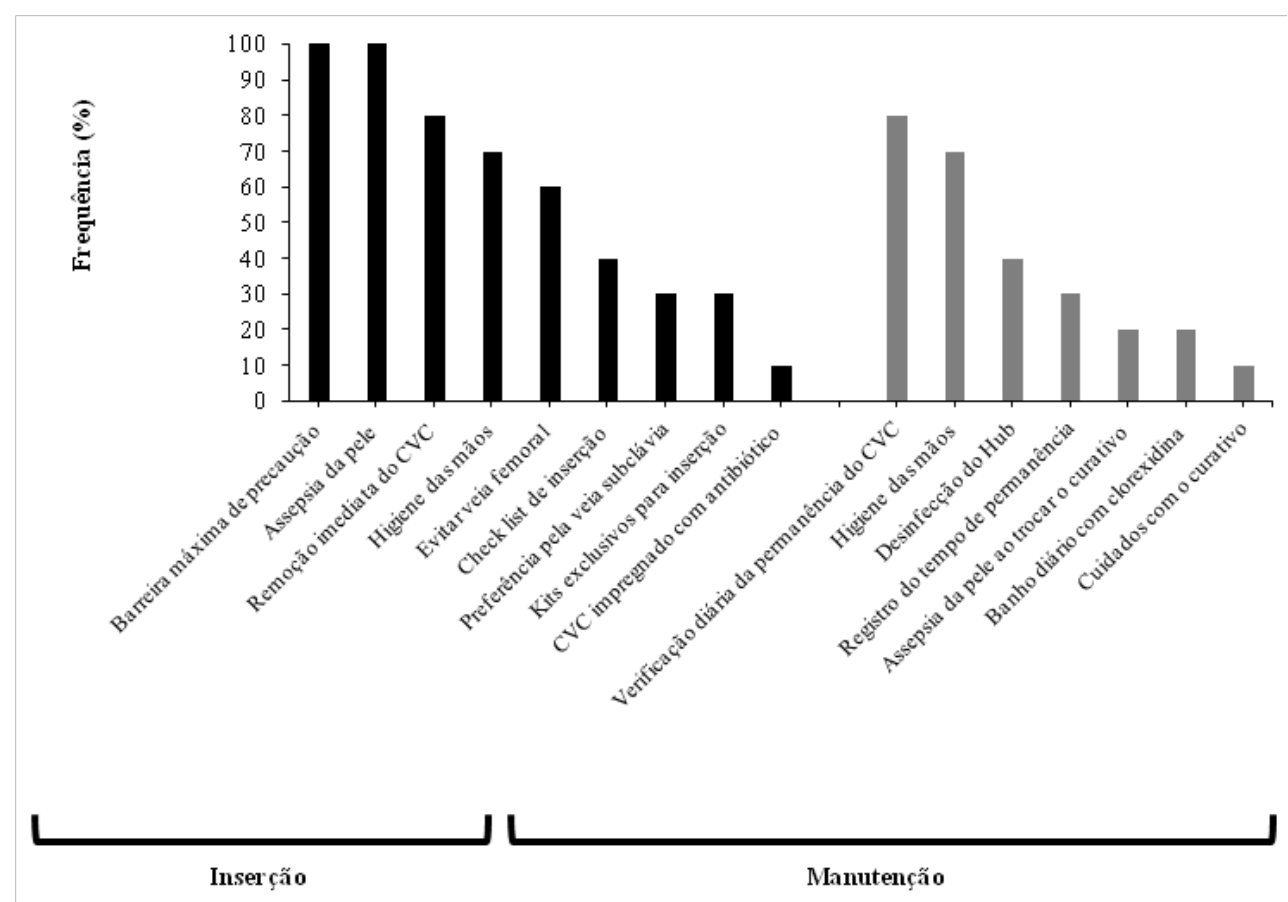

Figura 1. Frequência (\%) das medidas implementadas no período de intervenção dos estudos publicados, 2011-2016 
No período pós-intervenção em $100 \%$ dos estudos foi avaliado o impacto das estratégias implementadas sobre as taxas de infecção da corrente sanguínea relacionada ao CVC e disponibilizados feedback dos resultados e relatórios de vigilância à toda equipe.

As taxas de infecção da corrente sanguínea relacionada ao CVC nos períodos pré e pós-intervenção estão apresentadas na Figura 2.

Destaca-se ainda a relação entre a duração da intervenção e a ocorrência das infecções da corrente sanguínea relacionada ao CVC após o período de intervenção, representados na Figura 3.

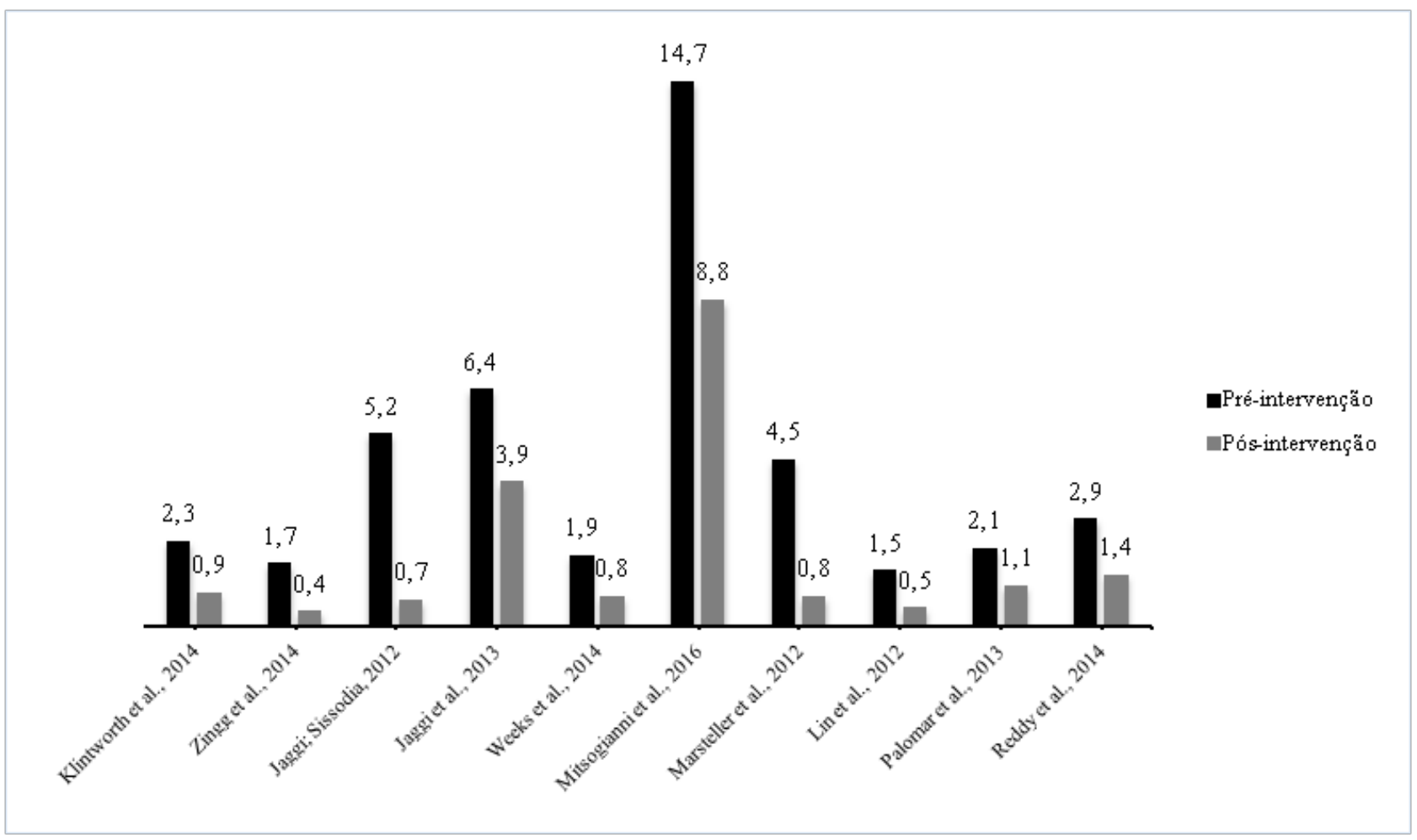

Figura 2. Taxas de infecção da corrente sanguínea relacionada ao CVC por 1000 CVC-dia nos períodos pré e pós-intervenção, 2011-2016

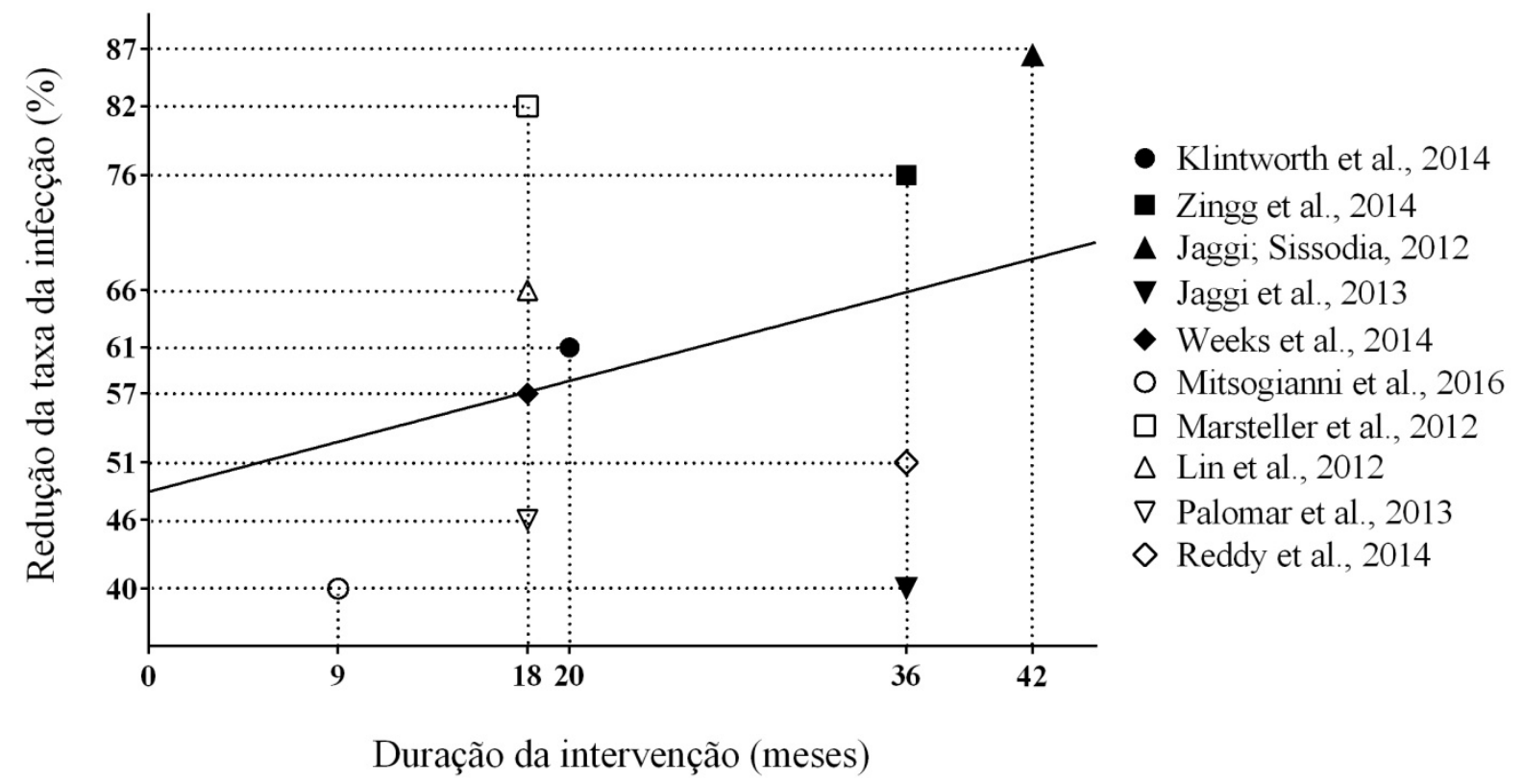

Figura 3. Relação da duração do período de intervenção com a taxa de redução da infecção da correte sanguínea relacionada ao CVC nos estudos analisados, 2011-2016 
Ao analisar os dados dos artigos que compararam a duração da intervenção com a ocorrência da infecção, evidencia-se uma correlação positiva entre o tempo em meses da intervenção e a porcentagem de redução das taxas de infecção. Pelo ajuste linear obtido na figura 3, a inclinação da reta mostra que a cada mês de intervenção há um aumento relativo de $0,48 \%$ na redução das taxas de infecção.

Ressalta-se também que em 100\% dos estudos analisados, o diagnóstico da infecção da corrente sanguínea seguiu os critérios do National Healthcare Safety Network (NHSN) e foram expressas como taxa de infecção por 1000 CVC-dia.

\section{DISCUSSÃO}

Foram encontradas nos estudos duas medidas que compuseram o período pré-intervenção, sendo o preparo da instituição e diagnóstico situacional e análise retrospectiva das taxas de infecção da corrente sanguínea relacionada ao CVC. A preparação da instituição e diagnóstico situacional são partes cruciais de uma estratégia multimodal bem-sucedida, na qual ajudam na obtenção de informações sobre as estruturas, recursos existentes, cultura da instituição, bem como os fatores limitantes e favoráveis à implementação para, posteriormente, adaptar as intervenções de acordo com o local ${ }^{22-23}$. Além disso, as informações obtidas sobre as taxas de infecção auxiliam em uma comparação entre os períodos pré e pós-intervenção, assim revelam a efetividade da intervenção no controle da infecção ${ }^{24}$.

O período de intervenção foi constituído pela implementação de bundles, no qual ocorreu por meio de treinamentos das equipes responsáveis pela manutenção e inserção do CVC. Os programas de educação continuada para os profissionais de saúde tem demonstrado eficácia na prevenção e redução da infecção relacionada ao CVC. Estudos demonstraram redução da infecção da corrente sanguínea em UTI em até $100 \%$ após a realização de treinamentos com a equipe multidisciplinar, nas quais foram abordadas as medidas recomendadas durante a inserção e manutenção do $\mathrm{CVC}^{25-26}$. Ademais, o treinamento pode ser um dos meios de desenvolver competências dos trabalhadores para se tornarem mais produtivos, criativos, inovadores e alcançar a excelência na qualidade dos serviços prestados, podendo também favorecer mudanças de comportamentos e atitudes. Ressalta-se ainda que os treinamentos devem ser dinâmicos e voltados, principalmente, para a sensibilização do profissional, com foco em normas e atitudes e não apenas à transmissão de informações ${ }^{27}$.

Dentre as medidas que foram implementadas e compuseram os bundles para prevenção da infecção da corrente sanguínea relacionada ao $\mathrm{CVC}$, as principais adotadas nos estudos foram a barreira máxima de precaução, assepsia da pele, remoção imediata do CVC sem indicação clínica para seu uso, higiene das mãos, evitar veia femoral e verificação diária da necessidade de permanência do CVC. Ressalta-se a importância da utilização dessas medidas para diminuir a contaminação microbiológica do cateter e subsequente risco de infecção. Exemplo da relevância da adoção dessas medidas foi visto em um estudo em UTI na Austrália, onde ao implementálas em um período de 18 meses, conseguiram reduzir em $60 \%$ a taxa de infecção da corrente sanguínea relacionada ao $\mathrm{CVC}^{28}$. Com a mesma estratégia, Burden et al. ${ }^{29}$, Allen et al. ${ }^{30}$ e Thom $\mathrm{t}$ al. ${ }^{31}$ observaram uma redução na taxa de infecção em UTI nos Estados Unidos em 61\%, 85\% e $70 \%$, respectivamente, após 4 anos da implementação. Além, do impacto econômico nos custos gerados por essa infecção, demonstrado em alguns estudos que conseguiram uma redução de US\$ 539.902,00 e US\$ 198.600,00 após implementar o bundle i2-33 $^{32}$

No período pós-intervenção em $100 \%$ dos estudos avaliaram o impacto das estratégias implementadas sobre as taxas de infecção da corrente sanguínea relacionada ao CVC e posteriormente realizaram um feedback dos resultados e relatórios de vigilância a toda equipe. Embora em muitos casos sejam negligenciados dentro das instituições de saúde, deve-se realizar a vigilância, notificação das infecções, feedback dos resultados obtidos e auditoria dos processos e resultados, pois esses auxiliam no processo de ensino-aprendizagem, fornece continuamente, informações relacionadas ao desempenho profissional e sobre as práticas institucionais, além de favorecer a melhoria no trabalho, a qualidade dos cuidados e segurança do paciente ${ }^{34-36}$.

Observou-se nos estudos uma redução das taxas de infecção da corrente sanguínea relacionada ao CVC após o período de intervenção. Ademais, percebeu-se uma correlação positiva entre o tempo da intervenção e a porcentagem de redução das taxas de infecção, sendo esse um ponto que merece destaque, visto que as principais ferramentas para reduzir a infecção da corrente sanguínea estão relacionadas aos programas de vigilância e de educação aos profissionais envolvidos na inserção e manutenção do CVC. No entanto, essas medidas são eficazes se os programas permanecerem ativos e contínuos, sendo também necessárias verificações periódicas para avaliar as lacunas na prática e estabelecer melhorias ${ }^{37}$.

\section{CONCLUSÃO}

Demonstrou-se que a estratégia multimodal ou multifacetada reduz a infecção da corrente sanguínea relacionada ao $\mathrm{CVC}$, independente da quantidade de medidas utilizadas no período de intervenção. No entanto, constatou-se nos estudos que um maior tempo de duração das intervenções proporcionou uma maior a redução da infecção. Por outro lado, os pontos comuns em todos os 
estudos foram a realização de treinamentos no período de intervenção independente das formas: palestra, simulação, discussão de casos ou workshop e os feedbacks e relatórios dos resultados aos profissionais no período pós-intervenção. Esses achados demonstram a importância de uma educação continuada para aumentar a adesão das equipes responsáveis pela inserção e manutenção dos

\section{REFERÊNCIAS}

1. Agência Nacional de Vigilância Sanitária. Infecção primária da corrente sanguínea: análise do indicador nacional das unidades de terapia intensiva brasileiras no ano de 2013. Bol Inf Seg Paciente Qualidade Serv Saúde (Brasília). 2014;4(8):1-23. Disponível em: https://www20.anvisa. gov.br/segurancadopaciente/index.php/publicacoes/item/ boletim.

2. World Health Organization (WHO). Report on the burden of endemic health care-associated infection worldwide. A systematic review of the literature. Geneva; 2011. Available from: http://apps.who.int/iris/ bitstream/10665/80135/1/9789241501507_eng.pdf.

3. Weaver SJ, Weeks K, Pham JC, Pronovost PJ. On the CUSP: Stop BSI: evaluating the relationship between central lineassociated bloodstream infection rate and patient safety climate profile. Am J Infect Control. 2014;42(10):S203-S208. doi: 10.1016/j.ajic.2014.05.020.

4. National Healthcare Safety Network (NHSN). Patient safety component manual. Atlanta; 2017. Available from: https:// www.cdc.gov/nhsn/pdfs/pscmanual/pcsmanual_current.pdf.

5. Mok Q. How I diagnose and manage catheter-associated blood stream infections. J Paediatr Child Health. 2015;25(5):24344. doi: http://dx.doi.org/10.1016/j.paed.2014.10.012.

6. Agência Nacional de Vigilância Sanitária. Avaliação dos indicadores nacionais de infecção relacionada à assistência ano de 2014 e relatório de progresso. Bol Inf Seg Paciente Qualidade Serv Saúde (Brasília). 2015;4(11):1-37. Disponível em: https://www20.anvisa.gov.br/segurancadopaciente/ index.php/publicacoes/item/11-boletim-informativoseguranca-do-paciente-e-qualidade-em-servicos-de-saude.

7. Agência Nacional de Vigilância Sanitária. Indicador Nacional de Infecção Hospitalar - Infecção Primária de Corrente Sanguínea Associada a Cateter Venoso Central: Análise dos dados das Unidades de Terapia Intensiva Brasileiras no ano de 2012. Bol Inf Seg Paciente Qualidade Serv Saúde (Brasília). 2013;3(5):1-22. Disponível em: https://www20. anvisa.gov.br/segurancadopaciente/index.php/publicacoes/ item/06-indicador-nacional-de-infeccao-hospitalar-infeccaoprimaria-de-corrente-sanguinea-associada-a-catetervenoso-central-analise-dos-dados-das-unidades-de-terapiaintensiva-brasileiras-no-ano-de-2012.

8. Salama MF, Jamal W, Mousa HA.; Rotimi V. Implementation of central venous catheter bundle in an intensive care unit in Kuwait: Effect on central line-associated bloodstream infections. J Infect Public Health. 2015;9(1):34-41. doi: 10.1016/j.jiph.2015.05.001.

9. World Health Organization (WHO). Guidelines on hand
CVC em relação às medidas de prevenção da infecção da corrente sanguínea. Isso evidencia claramente um maior compromisso destes profissionais, o que reflete na redução da infecção com relação direta ao tempo de duração da intervenção e, consequentemente, pode favorecer uma melhoria da qualidade da assistência prestada capaz de influenciar na segurança do paciente.

hygiene in health care. Geneva; 2009. Available from: http:// apps.who.int/iris/bitstream/10665/44102/1/9789241597906_ eng.pdf.

10. Zingg W, Pittet D. Central-line bundles need a multimodal implementation strategy. Lancet Infect Dis. 2016;16(6):6312. doi: http://dx.doi.org/10.1016/s1473-3099(15)00480-6.

11. Stetler CB, Morsi D, Rucki S, Broughton S, Corrigan B, Fitzgerald J, et al. Utilization-focused integrative reviews in a nursing service. Appl Nurs Res. 1998;11(4):1-11. doi: http://dx.doi.org/10.1016/s0897-1897(98)80329-7.

12. Klintworth G, Stafford J, O'Connor M, Leong T, Hamley $\mathrm{L}$, Watson $\mathrm{K}$, et al. Beyond the intensive care unit bundle: Implementation of a successful hospital-wide initiative to reduce central line-associated bloodstream infections. Am J Infect Control. 2014;42(6):685-7. doi:http://dx.doi. org/10.1016/j.ajic.2014.02.026.

13. Zingg W, Cartier V, Inan C, Touveneau S, Theriault M, GayetAgeron A, et al. Hospital-Wide Multidisciplinary, Multimodal Intervention Programme to Reduce Central Venous CatheterAssociated Bloodstream Infection. PLoS ONE. 2014; 9(4): e93898. doi: 10.1371/journal.pone.0093898.

14. Jaggi N, Rodrigues C, Rosenthal VR, Todi SK, Shah $\mathrm{S}$, Saini N, et al. Impact of an International Nosocomial Infection Control Consortium multidimensional approach on central line-associated bloodstream infection rates in adult intensive care units in eight cities in India. Int J Infect Dis. 2013;17(12):1218-24. doi: 10.1016/j.ijid.2013.07.007.

15. Weeks KR, Hsu HY, Yang T, Sawyer M, Marsteller JA. Influence of a multifaceted intervention on central line days in intensive care units: Results of a national multisite study. Am J Infect Control, 2014; 42(10): 197-202. doi: 10.1016/j. ajic.2014.06.003.

16. Mitsogianni M, Vasileiadis L, Parisi M, Tzanis G, Kampisiouli E, Psaroudaki $Z$ et al. A multifaceted intervention program to prevent bloodstream infection in an intensivecare unit running head: an intervention for the reduction of bacteraemia in ICU. Health Sci J. 2016;10(2):1-5. Available from: http:// www.hsj.gr/medicine/a-multifaceted-intervention-programto-prevent-bloodstream-infection-in-an-intensivecare-unitrunning-head-an-intervention-for-the.php?aid=8760.

17. Marsteller JA, Sexton JB, Hsu YJ, Hsiao CJ, Holzmueller $\mathrm{CG}$, Pronovost PJ, et al. A multicenter, phased, clusterrandomized controlled trial to reduce central line-associated bloodstream infections in intensive care units. Crit Care Med. 2012;40(11):2933-9. doi: 10.1097/CCM.0b013e31825fd4d8.

18. Lin DM, Weeks K, Bauer L, Combes JR, George CT, Goeschel CA, Lubomski LH, et al. Eradicating central 
line-associated bloodstream infections statewide: the Hawaii experience. Am J Med Qual. 2012;27(2):124-9. doi: 10.1177/1062860611414299

19. Palomar M, Álvarez-Lerma F, Riera A, Díaz MT, Torres F, Agra Y, et al. Impact of a national multimodal intervention to prevent catheter-related bloodstream infection in the ICU: the Spanish experience. Clin Invest. 2013;41(10):2364-72. doi: 10.1097/CCM.0b013e3182923622.

20. Reddy KK, Samuel A, Smiley KA, Weber S, Hon H. Reducing Central line-associated bloodstream infections in Three ICUs at a tertiary care hospital in the United Arab Emirates. Crit Care. 2014;40(12):559-66. doi: http://dx.doi. org/10.1016/s1553-7250(14)40072-2.

21. Jaggi N, Sissodia P. Repeated multimodal supervision programs to reduce the central line-associated bloodstream infection rates in an Indian corporate hospital. Healthcare Infect. 2013;19:53-8. doi: https://doi.org/10.1071/hi13030.

22. Organização Mundial da Saúde. Guia para implementação: um guia para a implantação da estratégia multimodal da OMS para a melhoria da higienização das mãos a observadores: estratégia multimodal da OMS para a melhoria da higienização das mãos. Brasília: Organização Pan-Americana da Saúde; Agência Nacional de Vigilância Sanitária. 2008. Available from: http://www.anvisa.gov. br/servicosaude/controle/higienizacao_oms/manual_para observadores-miolo.pdf.

23. Freire EMR, Martinez MR. Diagnóstico situacional: ferramenta de auxílio em gestão da qualidade. Rev Enferm UFPE. 2014;8(5):1405-12. doi: 10.5205/reuol.5863-505311-ED.0805201440.

24. Jardim, JM, Lacerda, RA, Soares, NDJD, Nunes, BK. Avaliação das práticas de prevenção e controle de infecção da corrente sanguínea em um hospital governamental. Rev Esc Enferm USP. 2013;47(1):38-45. doi: http://dx.doi. org/10.1590/S0080-62342013000100005.

25. Zingg W, Cartier V, Inan C, Touveneau S, Theriault M, Gayet-Ageron A, et al. Hospital-wide multidisciplinary, multimodal intervention programme to reduce central venous catheter-associated bloodstream infection. PLoS ONE. 2014;9(4):e93898. doi: 10.1371/journal.pone.0093898.

26. Wu PP, Liu CE, Chang CY, Huang HC, Syu SS, Wang $\mathrm{CH}$, Huang YC. Decreasing catheter-related bloodstream infections in the intensive care unit: interventions in a medical center in central Taiwan. J Microbiol Immunol Infect. 2012;45(5):370-6. doi: 10.1016/j.jmii.2011.12.016.

27. Leandro AIP, Branco ES. Importância do treinamento e desenvolvimento nos serviços de saúde. Rev Adm Hosp Inov Saúde. 2011;(6):64-9. doi: http://dx.doi.org/10.21450/rahis. v0i6.1018.

28. Burrell AR, McLaws ML, Murgo M, Calabria E, Pantle AC,
Herkes R. Aseptic insertion of central venous lines to reduce bacteraemia. Med J Aust Pyrmont. 2011;194(11):583-7.

29. Burden AR, Torjman MC, Dy GE, Jaffe JD, Littman JJ, Nawar F, et al. Prevention of central venous catheter-related bloodstream infections: is it time to add simulation training to the prevention bundle? J Clin Anesth. 2012;24(7):555-60. doi: 10.1016/j.jclinane.2012.04.006.

30. Allen GB, Miller V, Nicholas C, Hess S, Cordes MK, Fortune $\mathrm{JB}$, et al. A multitiered strategy of simulation training, kit consolidation, and electronic documentation is associated with a reduction in central line-associated bloodstream infections. Am J Infect Control. 2014;42(6):643-8. doi: 10.1016/j.ajic.2014.02.014.

31. Thom KA, Li S, Custer M, Preas MA, Rew CD, Cafeo C, et al. Successful implementation of a unit-based quality nurse to reduce central line-associated bloodstream infections. Am J Infect Control. 2014;42(2):139-43. doi: 10.1016/j. ajic.2013.08.006.

32. Sacks GD, Diggs BS, Hadjizacharia P, Green D, Salim A, Malinoski DJ. Reducing the rate of catheter-associated bloodstream infections in a surgical intensive care unit using the Institute for Healthcare Improvement Central Line Bundle. Am J Surg. 2014;207(6):817-23. doi: 10.1016/j. amjsurg.2013.08.041.

33. Longmate AG, Ellis KS, Boyle L, Maher S, Cairns CJ, Lloyd SM, Lang C. Elimination of central-venouscatheterrelated bloodstream infections from the intensive care unit. BMJ Qual Saf. 2011;20(2):174-80. doi: 10.1136/ bmjqs.2009.037200.

34. Flottorp SA; Jamtvedt G; Gibis B; McKee M. Using audit and feedback to health professionals to improve the quality and safety of health care. Geneva: WHO, European Observatory on Health Systems and Policies; 2010. Available from: http:// www.euro.who.int/_data/assets/pdf_file/0003/124419/ e94296.pdf.

35. Chesshyre E, Goff Z, Bowen A, Carapetis J. The prevention, diagnosis and management of central venous line infections in children. J Infect. 2015;71:S59-S75. doi: 10.1016/j. jinf.2015.04.029.

36. Cherry RA, West CE, Hamilton MC, Rafferty, CM, Hollenbeak CS, Caputo GM. Reduction of central venous catheter associated blood stream infections following implementation of a resident oversight and credentialing policy. Patient Saf Surg. 2011;5(1):15. doi: 10.1186/17549493-5-15.

37. Martínez-Morel HR., Sánchez-Payá J, Molina-Gómez MJ, García-Shimizu P, Román VG, Villanueva-Ruiz C, et al. Catheter-related bloodstream infection: burden of disease in a tertiary hospital. J Hosp Infect. 2014;87(3):165-70. doi: 10.1016/j.jhin.2014.04.008. 